

\title{
Los fundamentos del enfoque cualitativo en la innovación de la enseñanza geográfica
}

\author{
The foundations of the qualitative approach \\ in the innovation of geographical education
}

DOI: $10.7203 / D C E S .35 .12918$

\author{
José Armando Santiago Rivera \\ Universidad de los Andes (Venezuela) \\ asantia@ula.ve
}

\begin{abstract}
RESUMEN: En los avances paradigmáticos y epistemológicos del siglo XX, el enfoque cualitativo destaca como referente para construir el conocimiento social, entender la vigencia de los fundamentos tradicionales en la enseñanza geográfica y proponer aportes pedagógicos y didácticos en la innovación de su labor educativa. Eso determinó preguntar qué tarea cumplen los fundamentos del enfoque cualitativo en la innovación de la enseñanza de la Geografía. Al respecto, se realizó una consulta bibliográfica para estructurar un planteamiento que analiza el enfoque cualitativo en la enseñanza de la Geografía. A continuación, se describen aspectos derivados de una investigación que reivindica la manifestación de la subjetividad del docente, como opción demostrativa de su contribución en la innovación disciplinar y pedagógica.
\end{abstract}

Palabras Claves: Enfoque Cualitativo, innovación, enseñanza geográfica

\begin{abstract}
In the paradigmatic and epistemological advances of the $20^{\text {th }}$ century, the qualitative approach stands out as a reference for building social knowledge, understanding the validity of traditional foundations in geographical education and proposing pedagogical and didactic contributions in the innovation of its educational work. This led us pose the following question: What task do the foundations of the qualitative approach fulfill in the innovation of the teaching of Geography? In this regard, a bibliographical review was carried out to structure a methodology that analyzes the qualitative approach in the teaching of Geography. Likewise, aspects derived from an investigation that claims the manifestation of the subjectivity of the teacher, as a demonstrative option of its contribution in disciplinary and pedagogical innovation are described.
\end{abstract}

KEYWORDS: Qualitative Science, Innovation, Geographic Teaching

Fecha de recepción: julio de 2018 Fecha de aceptación: diciembre de 2018

Este artículo es producto de la investigación titulada "La investigación cualitativa en la enseñanza geográfica y la innovación didáctica de la práctica escolar cotidiana”, aprobada por el Consejo de Desarrollo Científico, Tecnológico, Humanístico y de las Artes (CDCHTA-ULA) (2017), de la Universidad de los Andes, Venezuela, bajo el Código: NUTA-H-405-16-04-B. 


\section{INTRODUCCIÓN}

El motivo de este análisis es destacar que durante el siglo XX, se desarrolló la permanente renovación epistémica; como situación poco comparable con otras etapas históricas pasadas (Mieres, 1996). Un hecho resaltable fue el declive del positivismo decimonónico y el afianzamiento del enfoque cualitativo en las ciencias sociales, de cuyos fundamentos derivaron otros enfoques para explicar la compleja dinámica social (Souto, 2016). En este acontecimiento se reivindicó al sujeto como actor de la realidad, por ser protagonista de los sucesos de la época y de la vida cotidiana, quien puede dar otra explicación a las circunstancias vivida, al expresar sus puntos de vista sobre los problemáticas del lugar (Rojas de Escalona, 2010).

Este aporte epistémico permitió a la investigación científica asumir la formación geográfica fundada en la reflexión crítica de los sujetos involucrados en el estudio. Igualmente interpretar la práctica escolar, en su finalidad educativa, el currículo establecido, los programas escolares de las asignaturas geográficas, las estrategias de enseñanza y de evaluación, en los criterios personales de docentes, estudiantes, autoridades escolares y los habitantes de la comunidad, como saberes factibles de innovar el acto educante, la gestión educativa y el modelo educativo.

Se trata de la ciencia naturalista, interpretativa y/o cualitativa, concebida como opción para gestionar el mejoramiento de la calidad formativa de la enseñanza geográfica. Esta orientación científica reivindica al ciudadano, dada su capacidad de motivación, curiosidad, creatividad y redescubrir lo real, como construir el conocimiento. El interés por su aplicación en la investigación, obedece al propósito de optimizar su tarea pedagógica y didáctica; actualizar la explicación del territorio, la organización del espacio y renovar la enseñanza y el aprendizaje en la práctica escolar cotidiana (Pulgarin, 2002; Gurevich 2009; González 2011, Santiago 2014 y Souto 2016).

Eso determinó formular la siguiente pregunta: ¿Qué tarea cumplen los fundamentos del enfoque cualitativo en la innovación de la enseñanza de la Geografía? Metodológicamente se consultó bibliografía sobre temas referidos a la explicación del enfoque cualitativo como opción epistémica para renovar la enseñanza de la Geografía. Finalmente, se demuestra la importancia del enfoque cualitativo, al exponer los hallazgos de una investigación que interrogó a docentes para descifrar las razones explicativas del uso didáctico de la televisión en la enseñanza geográfica.

\section{EL ENFOQUE CUALITATIVO}

En el inicio del siglo XX, los criterios para estructurar la verdad científica fueron establecidos por la orientación positivista, fundados en el mecanicismo, la linealidad y el funcionalismo, para avalar la validez y la confiabilidad del conocimiento. La veracidad derivó del desarrollo de experiencias estrictas, rigorosas y precisas, pues fue necesario asegurar lo valido determinó preservar la objetividad, sostenida en la neutralidad, la imparcialidad y el rigor. Así, la ciencia superó la especulación metafísica medieval con lo cierto, inobjetable e indudable.

En ese contexto, la explicación de la realidad geográfica se fundamentó en el determinismo, el historicismo, el posibilismo y el regionalismo (Souto, 2016) y los geógrafos asignaron prioridad al estudio físico del territorio, la influencia de las condiciones del entorno en la sociedad, la acción modificadora del grupo humano sobre el territorio, entre otros aspectos. Aunque desde mediados hasta el fin del siglo XX, la innovación paradigmática y epistemológica en la Geografía derivó en teorías la nueva Geografía, la Geografía de la Percepción, la Geografía radical, la Geografía humanística y la Geografía cultural (Âlvarez-Cruz, 2012).

Estas novedades epistémicas para concebir la verdad científica se apoyó en la aplicación de nuevas tecnologías, la retrospección para investigar la evolución del espacio y los fundamentos de la investigación acción participativa. En sus bases teóricas estuvo presente estudiar lo real, acorde con los cambios de la época, los avances disciplinares y los desafíos de los grupos humanos. Un aspecto que contribuyó a esos cambios epistémicos fueron las reflexiones sobre la observación; por 
ejemplo, se observa desde una teoría; toda observación es relativa al punto de vista del observador; cada observador distorsiona lo observado, lo real es una representación (Martínez, 1999).

Las acepciones citadas de la observación fueron evidencia de la anormalidad positivista, en su propósito por explicar los complejos y adversos eventos geográficos y sociales con el apoyo del modelo hipotético-deductivo. Las anomalías demostraron que la observación objetiva y lo sensualempírico, fueron poco confiables para autenticar la certeza y la veracidad, al reproducir lo observado con exactitud y se reivindicó los puntos de vista de los ciudadanos; por cierto, tradicionalmente cuestionados por la ciencia positiva debido al sesgo de los juicios de valor.

El hecho de observar desde una teoría y lo relativo del criterio personal sobre lo percibido, se entendió la realidad en su representación y significado. Así, se colocó en tela de juicio la exclusividad del paradigma positivo, ante la importancia epistémica asignada a la subjetividad. A fines del siglo XX, eso aportó el estudio de los problemas geográficos, al aplicar los fundamentos los fenomenológicos y etnográficos, ya empleados en estudios sociológicos y antropológicas.

En la Geografía científica se fortaleció la orientación humanística como opción para explicar la realidad con la versión centrada en la intervención activa, participativa y protagónica del investigador, además de acto indagador en esencia más humano y social. Se trata de la orientación cualitativa de la ciencia que aportó una verdad científica menos dogmática y determinista, al practicar razonamientos fundantes del pensamiento crítico y constructivo para: “...por una parte, ofrecer unas reflexiones que ayuden a adaptar una actitud científica como forma de pensar y de hacer que hemos de incorporar a nuestro modo de vivir; y por otra, destacar la necesidad de una reforma del pensamiento" (Ander-Egg, 2001a, p. 23).

Ante las nuevas condiciones sociohistóricas de fin de siglo, entender la realidad derivó en valorar la experiencia de los ciudadanos sobre los acontecimientos vividos, reivindicar el sentido común, la intuición y la investigación en la calle, al concebir una manera diferente de ver las cosas, innovar la percepción de los hechos, a la vez un pensar más ágil, ligero y abierto. Con estos cambios epistémicos: "A lo largo del siglo $\mathrm{XX}$, hemos vivido una transformación radical del concepto de conocimiento y de su respectivo concepto de ciencia. Estamos llegando a la adopción de un nuevo concepto de racionalidad científica, de un nuevo paradigma epistemológico" (Martínez, 2016, p. 63).

Con esta apertura, la ciencia se tornó expedita, emprendedora y atrevida, al facilitar inferencias analíticas sobre la realidad social, más allá de lo tradicional forma de pensar, teorizar y evaluar lo real. Uno cambios fue que las explicaciones geográficas deberían ajustarse a las condiciones sociohistóricas el momento. Allí, la tarea de deconstruir las situaciones estudiadas, supuso para la Geografía, realizar el análisis a partir de planteamientos analítico-críticos y constructivos de acento ideológico y político.

Ahora otra explicación derivó del estudio hermenéutico de lo real, mediante la acción interventora de quien lo investiga. Es interpretar el escenario donde los acontecimientos se desenvuelven en sus cambios, realizaciones y transformaciones, cuyo desciframiento demandó de opciones epistémicas contextualizadas en su propio territorio, pues: "Todas las realidades adquieren un significado de acuerdo al contexto en que son ubicadas. Es el contexto el que ofrece o asigna un significado a las cosas, al interactuar cada una de ellas con los elementos del mismo" (Martínez, 2016, 254).

Desde esta perspectiva, la Geografía y su enseñanza pudo considerar la posibilidad de iniciar un proceso de innovación ajustada a las circunstancias complicadas del inicio del nuevo milenio. Se trata de poder avanzar más allá de la tradición apreciada en la práctica escolar, dedicada a transmitir contenidos programáticos, a nivel de nociones y conceptos. Es educar en el aula de clase, evitar comprender el entorno inmediato, la época, a la vez que debilitar su tarea educativa, al preservar su condición de asignatura de cultura general.

Allí preocupa la limitación analítica a repetir la visión descriptiva de la realidad, resaltar los rasgos físico-naturales del territorio y enumerar detalles de las condiciones geográficas del lugar 
habitado. Es circunscribir el acto educante a la rutinaria cotidiana, percibir lo real como si fuera estático, entender lo sucedido con lo informado en la superficialidad mediática, ostentar criterios ajenos y mantener una postura contemplativa de los hechos. De esta forma se ha desnaturalizado su percepción del territorio, al desentenderse de las herramientas teóricas y metodológicas para desarrollar su análisis e interpretación.

Sin embargo, actualmente, en la Geografía y su enseñanza, se han propuesto iniciativas pedagógicas en el estudio de situaciones sobre la dinámica de la Geografía de las localidades, a partir de la obtención de los saberes empíricos de los ciudadanos. Eso ha significado: “...influir en los comportamientos de los actores sociales. No es sólo que se 'apliquen' luego de terminadas las investigaciones, lo cual sucede igualmente en las físico-naturales; sino que el hecho mismo de entrevistar a alguien o de hacerle una encuesta puede modificar su situación y acción frente al fenómeno estudiado (Follari, 2000, p. 19).

Estas experiencias han concebido el interrogatorio a ciudadanos, con la intención que faciliten su experiencia revelada en testimonios explicativos útiles al cambio de la problemática estudiada. Por tanto, esa manifestación expresa su sentido común e intuición, al explicar sus argumentos sobre el problema investigado. Es otra opción para interpretar las circunstancias geográficas de la localidad, además de la emitida por los expertos, donde generalmente priva lo teórico.

De esta manera, los ciudadanos pasan de ser espectadores de los hechos, a ser activos protagonistas en la interpretación de lo estudiado. Así, se facilita comprender lo que sucede desde otras lecturas analíticas y reflexivas, por lo menos heurísticamente más coherentes con lo real. Es la importancia epistémica de las anécdotas, los imaginarios, las representaciones, las costumbres, las tradiciones y las historias narradas por los habitantes; muchas de ellas, derivadas de la transmisión oral y otras, como expresión de la experiencia diaria.

Lo cierto es que la Geografía y su enseñanza encuentran en las internalidades de la subjetividad personal, saberes construidos en la convivencia colectiva del pensar y actuar social, como opción para mejorar la calidad formativa de sus procesos de enseñanza y de aprendizaje. Es la posibilidad de estructurar otras opciones pedagógicas y didácticas para renovar su tarea formativa, en la viva voz de los ciudadanos y, con eso, contribuir a su formación con un sentido humanizado. Es el acto pedagógico y didáctico direccionado con la acción investigativa centrada en el qué, por qué y para qué de los sucesos ambientales, geográficos y sociales.

Ya no es solo el acto descriptivo derivado del tratamiento estadístico, la única fórmula para obtener el conocimiento en la enseñanza de la Geografía, sino también es posible desarrollar el acto revelador de lo que piensan los ciudadanos sobre su realidad geográfica. Es asumir las vivencias, los pensamientos y las narraciones de los actores del objeto de estudio e implica para la escuela, asignar a la realidad geográfica su condición de escenario vivencial, entendido desde las percepciones de sus propios actores. Así, la Geografía y su enseñanza podrán facilitar a los ciudadanos, una visión humanizada de su realidad comunal, como constructo social e histórico.

\section{EL ENFOQUE CUALITATIVO EN LA ENSEÑANZA DE LA GEOGRAFÍA}

En las condiciones del momento histórico, desde mediados del siglo XX hasta el presente, se ha exhibido el extraordinario impulso de la ciencia y la tecnología, ante el impulso de la creatividad y la inventiva. Igualmente, es notorio el incremento de los adelantos tecnológicos, el desarrollo de la economía, la transformación de los medios de comunicación social. Aunque también son evidentes el atraso, la dependencia, la marginación, la pobreza y la exclusión. Por tanto, se trata de dos realidades muy distintas en cuanto su nivel de progreso y desarrollo.

Esta realidad ha motivado al sur a promover el debate sobre la descolonización, la identidad cultural y reivindicación de la cultura originaria, para contrarrestar la hegemonía del norte, pues para entender su realidad, las teorías científicas: “...fueron producidas en tres o cuatro países del 
Norte. Entonces nuestro primer problema para la gente que vive en el sur es que las teorías están fuera de lugar, no se adecuan realmente a nuestra realidades sociales" (De Sousa, 2009, p. 15).

Ante esta situación, vale citar entre las opciones epistémicas para redescubrir las complejas realidades geográficas contemporáneas, la investigación acción participativa, cuyo propósito ha sido estudiar lo real con la intervención vivencial de los investigadores como exigencia en la construcción del conocimiento. Se trata de indagar en procura del desciframiento del desenvolvimiento natural y espontáneo de lo estudiado, más allá de la objetividad de la ciencia hegemónica descriptiva.

Epistémicamente se ha colocado en el primer plano al observador, dada su capacidad empírica para opinar sobre lo real, desde su subjetividad, tal como ocurre en la fenomenología, la hermenéutica, la etnografía, el análisis del discurso y la investigación acción. En este caso, es oportunidad de otras explicaciones sobre las situaciones características de los territorios y la organización de los espacios, con la visión empírica de los ciudadanos allí involucrados. Significa la posibilidad de interpretar la realidad con la participación activa y protagónica del investigador y el interrogatorio a los habitantes en procura de un nuevo conocimiento geográfico.

Este aporte representó para la Geografía como ciencia, el hecho de reconocer la experiencia personal y reivindicar su lectura de los sucesos geográficos, pues: "El observador es parte de la realidad que observa. La llamada objetividad no es sino la determinación subjetiva que se hace de ella. Cada época, por así decirlo, ha construido su propia objetividad (Mieres, 1996, p. 169). Implica que la realidad existe y la investigación debe demostrar su presencia, para entender por qué sucede. El novedoso planteamiento es que quien observa lo real, es igualmente parte constitutiva de él.

Lo enunciado representa que: “...la investigación cualitativa se orienta hacia la construcción del conocimiento acerca de la realidad social y cultural a partir de la descripción e interpretación de las perspectivas de los sujetos involucrados" (Rojas de Escalona, 2010, p. 57). En el caso de la Geografía y su enseñanza, es la posibilidad de interrogar a los habitantes de una comunidad para luego desentrañar en sus criterios personales, opciones realizables de cambios significativos.

Otro aspecto interesante es que la investigación cualitativa: "es una actividad sistemática orientada a la comprensión en profundidad de fenómenos educativos y sociales, a la transformación de prácticas y escenarios socioeducativos, a la toma de decisiones y al descubrimiento y desarrollo de un cuerpo organizado de conocimientos" (Bautista, 2011, p. 71).

En la situación de la enseñanza de la Geografía, es estudiar el entorno inmediato a la escuela. Didácticamente es poner en práctica la indagación que amerita acercarse para conocer previamente al escenario geográfico, luego estructurar un plan de acción que oriente el desarrollo de las actividades pedagógicas y didácticas, para luego aperturar una discusión intencionada activada por los criterios personales de docentes, estudiantes y los protagonistas que facilitaron los datos.

Esta actividad posee un excelente valor pedagógico, porque el enfoque cualitativo de la ciencia, en su condición dialéctica y sistémica, permite concebir la explicación como una actividad integral desenvuelta en su propia dinámica geográfica. Por tanto, “...no se trata del estudio de cualidades separadas o separables, se trata del estudio de un todo integrado que forma o constituye una unidad de análisis y que hace que algo sea lo que es..." (Martínez, 2006, p. 128). En este nivel pedagógico, la enseñanza de la Geografía supera la visión fragmentada tradicional limitada a detallar la fisonomía físico-natural del territorio, lo ha establecido la Geografía descriptiva.

Con el enfoque cualitativo es posible asumir lo vivencial para estudiar los objetos geográficos en los lugares donde ocurren y explicar los sucesos en atención a los planteamientos personales de sus habitantes. Así se puede interpretar la realidad con una acción geográfica retrospectiva, cuyo propósito es descifrar la evolución geohistórica de la comunidad, desde su origen hasta el presente (González, 1997). Eso implica averiguar cómo era antes, cómo ha cambiado, qué factores han intervenido en sus cambios y cómo es en la actualidad. Se trata de un proceso constructivointerpretativo revelador de los hechos geográficos en sus diferentes momentos históricos. 
En esa dirección es descubrir o redescubrir las razones explicativas de los problemas geográficos e implica originar los cambios necesarios para invertir la situación estudiada (Baylina, 1997). De allí el interés en estudiar la vida cotidiana como tema de la enseñanza geográfica, al recurrir a la obtención de los puntos de vista de los ciudadanos, luego interpretar sus reflexiones, como base de un nuevo conocimiento. Lo relevante de esta acción es visibilizar significados y simbolismos, pues: "La realidad puede ser interpretada de varias maneras y la comprensión es dependiente de la interpretación subjetiva. La investigación cualitativa, basada en datos de narrativas y observaciones..." (Bueno, 2014: p. 106).

La intención es reconstruir con propósitos de innovación, la situación geográfica derivada del análisis hermenéutico. Es interrogar con la intervención científica, para capturar los criterios personales de quienes habitan el territorio. En consecuencia, en la enseñanza de la Geografía adquieren importancia didáctica las rutinas, experiencias, prácticas y simbolismos que caracterizan a la vida cotidiana comunitaria. Pero quizás lo más relevante es asumir sus sucesos en lo rutinario y habitual (Villegas y González (2011).

Al mismo tiempo es reivindicar la vulgaridad de lo cotidiano, pues con el enfoque cualitativo será posible examinar en forma más cercana, su desarrollo cotidiano, las diferentes maneras de comprender el escenario vivido en palabras del ciudadano como actor de sus propias vivencias. Es dar notabilidad a la manera personal de ver el mundo, la realidad y la vida, ahora requerido como aspecto innovador de la enseñanza de la Geografía, en la propósito de alfabetizar los ciudadanos sobre el tratamiento de su territorio y las dificultades ocasionadas por la irracionalidad revelada en la organización del espacio geográfico comunitario.

Esta labor, con los fundamentos de la investigación acción, facilita estudiar lo real con la intervención directa de quien investiga y visibilizar los puntos de vista de los ciudadanos. Es revelar la subjetividad para fortalecer la conciencia crítica y constructiva. Es conocer a partir de otra versión de la ciencia significativamente relacionada con el apremio de un hábitat humanizado y del respeto a lo natural.

\section{LA APLICACIÓN DEL ENFOQUE CUALITATIVO Y LA SUBJETIVIDAD DE DOCENTES EN LA ENSEÑANZA GEOGRÁFICA}

En los estudios sobre la innovación de la enseñanza de la Geografía, desde mediados del siglo XX, hasta la actualidad, ha prevalecido la indagación sobre qué se enseña y cómo se enseña. Allí, se ha podido apreciar las críticas sobre la vigencia de los fundamentos de la geografía descriptiva y la pedagogía tradicional, el estudio de los rasgos físicos-naturales del territorio y la vigencia de la orientación transmisiva de contenidos programáticos.

Sin embargo, una experiencia significativa ha sido visitar las instituciones escolares con la aplicación de los fundamentos del enfoque cualitativo, estudiar lo que ocurre habitualmente en las aulas escolares de la enseñanza geográfica e identificar testimonios verbales y/o escritos posibles de estructurar un conocimiento coherente sobre la realidad escolar. "De ahí que sea preciso penetrar en los factores más profundos, que a veces están ocultos por una cosmovisión implícita, a la que le denominamos representación social" (Souto, 2016, p. 100).

En tal sentido, las investigaciones ha sido direccionadas en procura de significados, representaciones, concepciones e imaginarios, emitidos con la responsabilidad personal al manifestar evidencias derivadas de la experiencia vivida, tal y cómo se ha desarrollado (Ander-Egg, 2001b). Esta labor científica se certifica al revelar una verdad, en muchos casos superflua, redundante e insustancial, pero confirmante de la práctica auténticamente sucedida.

Esta innovación epistémica ha permitido acceder al docente de Geografía, quien expresa su criterio sobre su práctica pedagógica, al ser interrogado con la aplicación de la orientación cualitativa de la ciencia, en la perspectiva fenomenológica. En consecuencia, con fines demostrativos, se citan las respuestas dadas por docentes en una investigación, cuyo propósito fue 
conocer los puntos de vista de docentes de Geografía sobre el uso didáctico de la televisión, en el intento de mejorar su calidad formativa (Santiago, 2014).

Metodológicamente ese estudio asumió el enfoque cualitativo, pues: “...produce datos descriptivos, en las propias palabras de las personas, habladas o escritas y la conducta observable (Rodríguez, Gil y García, 1999, p. 87). Por tanto, se pretendió obtener, ante la formulación de interrogantes, la narración de opiniones de docentes sobre la enseñanza de la Geografía con la aplicación didáctica de la televisión en la escuela (Hernández, 1998).

El estudio consideró la selección de los docentes participantes de manera intencional, quienes fueron elegidos por laborar en instituciones públicas de Educación Secundaria, en la ciudad de San Cristóbal, estado Táchira, Venezuela. La escogencia tomó en cuenta su formación profesional (docentes de Geografía y Ciencias de la Tierra, Geografía e Historia), facilitadores de las asignaturas de Geografía General, Geografía de Venezuela y Geografía Económica de Venezuela; sus años de servicio en la docencia, su experiencia en la enseñanza geográfica y su aprobación voluntaria para participar en la experiencia investigativa.

En efecto, colaboraron seis (6) educadores codificados, por ejemplo (D01), con la letra D y el número del procesamiento de sus respuestas. A ellos se aplicó un cuestionario de preguntas abiertas elaboradas luego de la revisión del problema planteado y de las bases teóricas del estudio. Eso para orientar la obtención de los datos desde la teoría hacia la práctica. El desarrollo del estudio se cumplió con las siguientes actividades:

a) Ante la formulación de la interrogante del estudio, se hizo la revisión de bibliografía relacionada con el objeto de la investigación, en consulta a artículos y libros en la biblioteca de la institución, en google académico, en www.saber.ula.ve., entre otros.

b) Se redactó el proyecto y se presentó a las instancias de la Universidad.

c) Una vez aprobado se aplicó el instrumento estructurado con preguntas elaboradas a partir de los fundamentos teóricos del estudio, en lo relacionado a la televisión, la Geografía desde la televisión y la enseñanza de la Geografía de la Televisión.

d) Previamente se realizó un estudio piloto con el propósito de evaluar las preguntas a formular en el cuestionario.

e) Los docentes respondieron el cuestionario de preguntas abiertas en su centro de trabajo.

f) Se recolectó el instrumento y se procedió a la codificación de cada uno.

g) Luego se procedió a leer, clasificar y codificar las respuestas.

h) Se obtuvo las subcategorías y, desde allí, las categorías.

i) A continuación, se procedió a triangular los datos obtenidos, con lo planteado por los expertos (Arias, 2006). Esta actividad fue determinante para preservar la objetividad requerida por estudios de esta naturaleza (Rodríguez, Gil y García, 1999).

Entre los hallazgos del estudio, se citan los siguientes:

4.1. El mundo contemporáneo es complicado. "Vivimos en un escenario adverso, en crisis y muy confuso" (DO2). "El mundo se nos presenta desarrollado, agitado y violento. En pocas palabras, el mundo que yo percibo es violento, lleno de incertidumbres". (DO1). "El mundo actual está en crisis: crisis económica, explosión demográfica, conflictos internacionales, deterioro ambiental, escasez de agua potable, entre otros". (DO6). "El mundo actual lo percibo cambiante, con una gran cúmulo de información que mantiene a los habitantes al día con todas las noticias ocurridas". (DO4). "Pienso que esta realidad tan complicada debe ser motivo de atención por la enseñanza de la geografía" (D03).

Con los aspectos descritos, el docente de Geografía involucrado en el estudio, reveló que gracias a la televisión, puede apreciar el momento histórico como época compleja, enrevesada, incierta, confusa, cambiante, con adversidades económicas, demográficas, geopolíticas y la problemática ambiental. Por tanto, recomienda prestar atención hacia esta complicada realidad en la enseñanza geográfica. Es llamativo que antes del estudio, se propuso educar para la complejidad 
(Alanis, 1999) y también se cuestionaba que los temas del mundo globalizado, no fuesen motivo de estudio en la enseñanza geográfica (Hollman, 2008).

4.2. La televisión es un medio para explicar el mundo actual. "Pienso que si los docentes utilizáramos la televisión, veríamos el mundo mejor que como lo enseñamos en clase" (DO5). "A1 mundo lo percibo complejo y desigual de acuerdo a lo observado en la televisión" (DO1). "Con ver la televisión podemos observar cualquier acontecimiento que se está presentando en cualquier sitio del planeta" D02). "Nuestra vida cotidiana está cambiando de manera radical ya que el avance de la tecnología nos ha hecho todo este escenario social donde nosotros como actores de esta película, estamos ante la necesidad de hacer un buen papel" (DO4). "Se impone ver de una manera diferente a la televisión, pues enseña muchas cosas importantes para la enseñanza de la geografía" (DO6).

En estas descripciones, se infiere que en la opinión de los docentes, gracias a la televisión, tienen una mejor percepción del mundo contemporáneo, debido a la información que facilita y recomiendan su uso didáctico en la enseñanza geográfica. Aunque ya se proponía que el proceso formativo debería prestar atención a los medios de comunicación social (Pérez-Esclarín, 2002), e igualmente se opinaba que ante el nuevo contexto cultural, se imponía renovar la enseñanza para descifrar la realidad propuesta por la acción mediática (Rodríguez, 2008).

4.3. La televisión es un excelente medio didáctico. "Personalmente pienso que en la realidad geográfica se están manifestando nuevos temas, problemas, inquietudes y retos. Es decir, debemos ver la geografía desde la televisión con un sentido real de los problemas que nos afectan a diario" (DO2). "Pienso que con el uso didáctico de la televisión la enseñanza de la geografía sería diferente" (DO3). "La televisión facilita buenos programas para mejorar la acción didáctica de la enseñanza de la geografia" (DO4). "El docente debe estar preparado para saber qué enseñar, cómo enseñar y con qué enseñar, porque el alumno se encuentra bien informado" (DO1). "Como persona tengo acceso a la Tv por cable, a la prensa, la radio y continuamente realizo talleres donde crezco como docente y como persona lo que me conecta con lo que pasa en el mundo" (DO6).

Los docentes involucrados en el estudio destacan la importancia didáctica de la televisión en la enseñanza de la Geografía. Entre los motivos, resaltan que ante la dificultad para enseñar los temas del mundo globalizado, la televisión ofrece programas interesantes sobre temas geográficos, además informa a los estudiantes. Ante lo planteado propuso la Geografía de la Televisión como la oportunidad pedagógica y didáctica de explicar la complicada realidad ambiental, geográfica y social, desde la práctica escolar cotidiana (Santiago, 2002).

4.4. La televisión puede mejorar la calidad formativa de la enseñanza geográfica. "Me está preocupando que los problemas del mundo actual se están tocando muy superficialmente en el aula de clase.” (DO1). “¿Cuántos programas geográficos podríamos utilizar, si por lo menos los liceos tuvieran un televisor? Seria fabuloso ya que es más difícil sacar a los alumnos al campo que mantenerlos observando y comentando su realidad" (DO3). "Lo importante de la televisión en la enseñanza de la geografía es que ofrece programas muy útiles para enseñar en el aula de clase" (DO6). "El docente enseña geografía debe dar a conocer los graves problemas que hoy día vive el mundo entero, es decir, orientados para que vivan el complejo momento" (DO2). "Un cambio muy efectivo es dar a la televisión el reconocimiento a su valor didáctico cuando se pretende mejorar la enseñanza de la geografía" (DO5). "Pienso que en el aula de clase, con los recursos de que disponemos, no podríamos enfrentar las consecuencias que nos genera la globalización. Para ello tenemos que salir del aula y enfrentar al alumno con las consecuencia que ella nos trae y eso se logra a través de la investigación." (DO4).

Ante la preocupación por el tratamiento pedagógico y didáctico de los problemas geográficos de manera muy superficial, los docentes valoran la importancia de la televisión en el aula. Este medio divulga situaciones útiles en lo didáctico para enseñar los eventos del mundo globalizado, al 
permitir identificar los problemas, estimular la investigación y reconocer que su uso puede mejorar la enseñanza de la Geografía. También, ante la exigencia de diversificar los recursos didácticos en el aula de clase, la televisión permite explicar los contenidos programáticos desde otras opciones pedagógicas (Souto y Gurevich, 2008).

4.5 La geografía de la televisión facilita el acercamiento a la realidad geográfica, porque: "Facilita trabajar temas de actualidad, discutiendo sobre acontecimientos del mundo, utilizar la imaginación para atacar la complejidad que vive la sociedad hoy día" (DO4). "Ya no solamente se habla de los temas tradicionales como la geografía rural o geografía urbana, sino de una geografía global" (DO1). "Estos cambios en la enseñanza de la geografía indican que debemos ser más investigadores, para hacer una enseñanza más relevante y coherente a las necesidades de los estudiantes" (DO3). "Los muchachos hoy día tienen acceso a una serie de ventajas (Tv por cable, Internet). Esto pone a su disposición más información, la cual, a veces, los lleva a intervenir en el desarrollo de la clase y plantear interrogantes sobre diferentes temas, como dato curiosos" (DO6). "Pienso que si utilizáramos a la televisión en el aula de clase, la enseñanza de la geografía nos facilitaría entender el mundo vivido" (DO2). "Pienso que para mejorar la enseñanza de la geografía es indispensable: a) tener computadoras, b) Acceso a la televisión y c) Acceso a la prensa" (DO5).

Para el punto de vista de los docentes consultados, la Geografía de la Televisión permite tratar temas de actualidad geográfica global y motivar su investigación. Eso facilita proponer una enseñanza coherente a las necesidades formativas de comprender los eventos geográficos y entender el mundo vivido. Según Ferres (1994), eso obliga al educador a estar informado sobre los temas expuestos en la televisión, al igual que revisar su condición de espectador, evitar la alienación y el acriticismo. Los aspectos descritos representan una valiosa contribución pedagógica al mejoramiento de la calidad formativa de la enseñanza de la Geografía; en especial, el aporte de testimonios que reivindican la importancia de los saberes experienciales, pues desde allí, se pueden realizar inferencias para justificar versiones factibles de promover aportes innovadores a la Geografía escolar.

Por cierto, en forma reiterativa se ha reivindicado la importancia de la televisión como recurso audiovisual, por apoyarse en imágenes y vivencias para exponer acontecimientos geográficos y propiciar explicaciones con argumentos pertinentes y coherentes sobre las temáticas y problemáticas geográficas divulgadas (Ferrés, 1994; Pulgarin, 2002; Santiago, 2002; Vera Aranda y Valero Palomo, 2005; García, 2006; Souto y Gurevich, 2008; Gurevich, 2009 y González, 2011). Con la Geografía de la Televisión se trata de popularizar situaciones originadas por la relación de los grupos humanos con la naturaleza, con el apoyo de la tecnología audiovisual donde se dificulta distinguir el aditivo simulador de lo artificial. Aunque no se evita visualizar el sentido imparcial y neutral del hecho, simulado con la espectacularidad y el atractivo, pues su misión es capturar al espectador pasivo, aunque capaz de elaborar un punto de vista sobre lo percibido.

\section{CONSIDERACIONES FINALES}

Ante las problemáticas características del mundo contemporáneo, la enseñanza de la Geografía debe mirar hacia otras opciones pedagógicas y didácticas que aporten una formación educativa coherente con las complejas situaciones que afectan a la calidad de vida de la sociedad. Es educar para entender y transformar, no solo la realidad, sino también la conciencia de los ciudadanos. En esa dirección, supone alfabetizar con fundamentos científicos y educativos, cuya epistemología genere confianza al desenvolvimiento de la investigación.

Es necesario reconocer que en la enseñanza de la Geografía se ha descuidado el tratamiento didáctico del estudio de las condiciones geográficas de la época y de la comunidad, ante la vigencia del modelo transmisivo tan arraigado en su práctica pedagógica. Su persistencia da origen a una 
acción pedagógica precaria, frágil e inconsistente, poco responsable porque privilegia la formación intelectual del ciudadano espectador neutral y apático. Explicar razones sobre esta extraordinaria debilidad formativa implica proponer la enseñanza geográfica fundada en conocimientos y prácticas del enfoque cualitativo, cuya labor formativa debe pretender:

a) Asumir las condiciones del momento sociohistórico como el escenario de cambios y transformaciones geográficas del mundo globalizado. Eso implica entender la época y sus acontecimientos en la perspectiva de los ciudadanos.

b) Aproximarse a las situaciones geográficas del mundo inmediato, con el propósito de descifrar la vida cotidiana, desde la experiencia adquirida por los habitantes de la comunidad.

c) Considerar la explicación de los acontecimientos del lugar con la participación activa y protagónica de la colectividad, al aplicar los fundamentos de la investigación cualitativa para obtener la subjetividad colectiva.

d) Facilitar la posibilidad de inmiscuirse en la comprensión del objeto de estudio, a partir el sentido común, la intuición y la investigación en la calle, como opciones naturales y espontáneas para conocer lo real desde los puntos de vista de los ciudadanos.

e) Utilizar la investigación cualitativa con el propósito de innovar la transmisividad tradicional, por una práctica escolar cotidiana, centrada en el desarrollo de un acto educante estimulador de la elaboración del conocimiento.

Los aspectos descritos deben ser considerados como desafíos en las propuestas para innovar las orientaciones formativas de la Educación Geográfica. Se trata de colocar en el primer plano la necesidad que la enseñanza de la Geografía, contribuya a formar la conciencia crítica y constructiva, manifestada en comportamientos de sentido humano y fortalecedor del valor auténtico de lo social.

Por tanto, se impone analizar la compleja situación de la práctica pedagógica tradicional. Eso supone reivindicar la orientación cualitativa de la ciencia, en lo referido a valorar la subjetividad personal, la experiencia derivada de la participación, el protagonismo social y la aplicación didáctica de la investigación acción. Allí, el logro significativo debe ser educar la conciencia crítica y constructiva en los educandos sobre la complicada realidad de su momento histórico.

\section{Referencias}

Alanis F., L. (1999). Educar para la complejidad: contenidos de enseñanza y movimiento sociales. La influencia de la sociedad civil en el curriculum de ciencias sociales. Un curriculum de ciencias sociales para el siglo XXI. ¿Qué contenidos y para qué? Sevilla: Díada S.L.

Álvarez-Cruz, P. (2012). Enfoques de la ciencia geográfica y su proyección en el proceso de enseñanza-aprendizaje. Varona. Revista Científico-Metodológica, 54, 58-64.

Ander-Egg, E. (2001a). Globalización. El proceso en el que estamos metidos. Córdova (Argentina): Editorial Brujas.

Ander-Egg, E. (2001b). Métodos y técnicas de investigación social I Acerca del conocimiento y del pensar científico. Buenos Aires: Grupo Editorial Lumen SRL.

Arias, F. (2006). El proyecto de investigación. Introducción a la metodología científica. Caracas: Editorial Episteme.

Bautista C., N. P. (2011). Proceso de la investigación cualitativa. Epistemología, metodología y aplicaciones. Bogotá: Manual Moderno.

Baylina Ferré, M. (1997). Metodología cualitativa y estudios de geografía y género. Documents d'anàlisi geogràfica, 30, 123-138. 
Bueno Cuadra, R. (2014). Una nota sobre complejidad y paradigma cualitativo. Liberabit. Revista de Psicología, 20(2). Recuperado de http://www.redalyc.org/articulo.oa?id=68632617015. [12 de marzo de 2018].

De Sousa Santos, B. (2009). Una epistemología del Sur. La reinvención del conocimiento y la emancipación social. México: CLACSO y Siglo XXI.

Ferrés, J. (1994). Televisión y educación. Barcelona: Ediciones Paidós Ibérica, S.A.

Follari, R. (2000). Epistemología y sociedad. Rosario: Homo Sapiens Ediciones.

García, E. (2006). Una televisión para la educación. La utopía posible. Barcelona: Editorial Gedisa.

González O., J. L. (2011). Educación y geografía para una crisis. Didáctica Geográfica, 12, 11-16.

González Rey, F. (1997). Epistemología cualitativa y subjetividad. La Habana: Editorial Pueblo y Educación.

Gurevich, R. (2009). Territorios y lugares del mundo hoy: notas para su enseñanza. Revista Digital 12 (ntes). 4.

Hernández R., G. (1998). Paradigmas en psicología de la educación. México: Editorial Paidós Mexicana.

Hollman, V. C. (2008). La globalización en la geografía escolar: Continuidades y rupturas en la construcción geográfica de un contenido. Biblio $3 W$ Revista Bibliográfica de Geografía y Ciencias Sociales. XIII(803).

Martínez Migueles, M. (2016). El conocimiento y la ciencia en el siglo XXI y sus dificultades esterognósticas. Segunda Edición. Caracas: Editorial Trillas.

Martínez Migueles, M. (1999). La nueva ciencia. México: Editorial Trillas, S.A.

Mieres, F. (1996). La revolución que nadie soñó o la otra posmodernidad. Caracas, Editorial Nueva Sociedad.

Pérez-Esclarín, A. (2002). Educar en el Tercer Milenio. Caracas: San Pablo.

Pulgarin, R. (2002). Ciencias Sociales en la Educación Básica. Lineamientos Curriculares. Bogotá: Ministerio de Educación Nacional/Cooperativa Editorial Magisterio.

Rodríguez Domenech, M. Á. (2008). Una enseñanza nueva en una cultura nueva. El caso de la geografía en el Bachillerato. Actas del X Coloquio Internacional de Geocrítica. Universidad de Barcelona. Barcelona.

Rodríguez G., G., Gil F., J. y García J., E. (1999). Metodología de la investigación cualitativa. Archidona (Málaga): Ediciones Aljibe, S. L.

Rojas de Escalona, B. (2010). Investigación cualitativa. Fundamentos y praxis. Caracas: Fondo Editorial De la Universidad Pedagógica Experimental Libertador.

Santiago Rivera., J. A. (2002). La geografía de la televisión y la enseñanza de la geografía. Revista Educere, 6(19), 268-275.

Santiago Rivera, J. A. (2014). La geografía de la televisión desde la experiencia pedagógica de los docentes. Revista Acción Pedagógica, 23, 106-113.

Santiago Rivera, J. A. (2016). Los cambios geohistóricos, la enseñanza geográfica y la educación secundaria en Venezuela, desde el siglo XIX al XX. Revista Educare, 20(1), 57-80.

Souto González, X. M. (2016). La investigación cualitativa y la innovación didáctica en geografía. El trabajo cualitativo como recurso didáctico en geografía. Actas XI Congreso Nacional Didáctica de la Geografía.

Souto, P. y Gurevich, R. (2008). Las representaciones de la geografía en la tv: Una aproximación al tema a través de la opinión de padres de alumnos de nivel medio. Question, Revista especializada de periodismo y comunicación, 1(20). Recuperado de http://perio.unlp.edu. ar/ojs/index.php/question/article/viewFile/697/60 [15 de marzo de 2018].

Vera Aranda, A. L. y Valero Palomo, M. del P. (2005). La utilización didáctica de la televisión para el aprendizaje de la geografía. Comunicar. Revista científica iberoamericana de 

$\begin{array}{lcccc}\text { comunicación } & y & \text { educación, } & \text { 25(2). } & \text { Recuperado } \\ \text { http://dialnet.unirioja.es/serviet/articulo?codigo=2929191 [20 de marzo de 2018]. }\end{array}$

de

Villegas, M. M. y González, F. (2011). La investigación cualitativa de la vida cotidiana. Medio para la construcción de conocimiento sobre lo social a partir d lo individual. Revista Psicoperspectivas. Individuo y Sociedad, 10(2), 35-59.

\section{CÓMO CITAR ESTE ARTÍ́CULO}

Santiago Rivera, J. A. (2018). Los fundamentos del enfoque cualitativo en la innovación de la enseñanza geográfica. Didáctica de las ciencias experimentales y sociales, 35, 33-44. DOI: 10.7203/DCES.35.12918. 